\title{
Effects of Cultural Beliefs on Cancer Prevalence
}

\author{
Xiaoyuan Jin \\ School of Public Health \\ University of California Irvine \\ Irvine, American
}

\begin{abstract}
This paper talks about the cultural factors that influence the infection and the treatment of cancer. In the paper, the author provides some examples of people of different races all around the world to prove the fact that cultural beliefs have influence to the diseases. Moreover, the paper indicates the problems lying in the diseases treatment that are governed by cultural difference, and the author provides some suggestions for the problems.
\end{abstract}

Keywords—cultural beliefs; cancer prevalence; challenges

\section{INTRODUCTION}

Health is significantly determined by a diverse array of factors ranging from genetic inheritance, access to quality care, personal behavioral choice, external environment, and other acculturated factors. ${ }^{1}$ A vast research on the association between cancer and cultural factors has been developed with different cultural constructs explored on their potential impact of the disparities, prevalence and etiology of this disease. For instance, it should be identified that the outset that these cultural variables can be conceptualized to influence cancer illness at multiple levels - with some research focusing on pain disparities across different cultural groups while other focus on the effects of cultural beliefs and practices on its prevalence and treatment interventions., Again, the detrimental health effect of growing in a family deeply rooted to cultural beliefs may be potentiated if the family resides in disadvantaged community where culture is given a weighty consideration than the westernized treatments. $^{3}$

A survey by the International Agency for Research on Cancer, 2014, identified that cancer accounts for about $12 \%$ of mortality rates across the globe. ${ }^{4}$ Ultimately, IARC suggested that the cancer myths and acculturated stigma and beliefs were salient pertinent issues that must be addressed, though the varied across the cultural groups. Notably, formulation of cancer stigmas is based on the different cultural belies that the people in a particular group tie to cancer. ${ }^{3}$ Such stigmas, as well as, beliefs may be challenging when it comes to developing treatment and cancer prevention health programs. Ultimately, research has tied stigma to silencing effects leading to poor cancer treatment especially the access to modernized cancer screening and treatment services. ${ }^{2,3}$

On a different note, cultural studies reveal that in the US society the African-Americans or the blacks had the highest statistics of cancer by 2013 , followed by the Hispanics and then the Indian - which all are minority groups identified to have strong cultural backgrounds. ${ }^{6-7}$ A survey by the National Cancer Institute found that prostate cancer has shown disproportionate burden across the various ethnic groups in America with a more weight on the blacks. Regardless of, genetic variance issues explaining such disproportionate, cultural beliefs and taboos have been linked with unhealthy cancer practices especially in seeking for early treatment and screening for early diagnosis.

Similarly, prostate cancer had similar patterns with NCI reporting that between 2000 and 2014, both the Americans and the Asians reported the highest incidence and mortality rates. The blacks had 255.5 incidence rate and $62.3 \%{ }^{7}$ mortality rates with the Asians having 96.5 incidence and $11.3 \%$ mortality rates. ${ }^{7}$ However, the whites had the lowest cancer-related deaths reported as $25.6 \%$ of the total mortalities for prostate cancer deaths. ${ }^{7}$ The chances that an African dies of either of the cancer types are higher as compared to any other ethnic groups. ${ }^{8}$ With significant evidence tied to health disparities and racial segregation in American health care systems, cultural beliefs have been identified to play a big part in shaping these statistics. ${ }^{8}$ This paper focuses identifying and exploring the effects of cultural beliefs on the prevalence of cancer to help understand the relationships between the two variables.

\section{CONCEPTUAL ISSUES}

\section{A. The Problem-How Cultural Beliefs Affect Cancer Prevalence}

While health care systems are trying to achieve a balanced scorecard in cancer prevention, the concept of cancer and culture still need attention. ${ }^{9}$ Many of the epidemiologic research statistically demonstrate insufficient explanatory evidence on the social, biologic and ethnic/racial differences realized with cancer morbidity, incidence, quality of life and mortality. ${ }^{9}$ Ideally, regardless the efforts by the health care systems to impart cultural competence knowledge on its practitioners, their goals of minimizing disparities appear to have fallen apart, with the issue of culture remaining poorly understood in cancer diagnosis, treatment and prevention. ${ }^{9,10}$ Every culture has its own beliefs regarding to the treatment methods, health care provision and the disease itself, implying that different cultural groups have distinct and different perceptions on cancer risks, screening and diagnosis techniques, as well as, 
the trust on the institutions and professionals carrying out cancer therapies. ${ }^{11}$

To add on this, cultural beliefs have a striking potential in explaining the health care disparities in cancer patients ranging from health care access to decisions on treatment alternatives available for cancer. ${ }^{11,12,13}$ Ideally, cultural groups may have indifferent perceptions and beliefs when it comes to radiology treatments or surgeries for cancer patients - beliefs that might come from religious practices or traditional remedies which discourage modernized treatment methods. ${ }^{11}$ Additionally, other cultural minority groups such as the Hispanics and the blacks are identified to rely heavily on high proteins and fatty diets - and part of these proteins are susceptible to cancerous cells. ${ }^{13}$

On the other hand, when it comes to issues related to genetic or biological etiology, cultural beliefs may not accredit or acknowledge the ideas that cancer is inherited while other beliefs on different therapies are unhealthy. ${ }^{3}$ Lastly, some ethnic minority groups may have the beliefs that they are poor, underserved and disadvantaged implying that they would be less concerned for securing health education or putting extra effort to access the Medicaid or Medicare services to access health insurance for cancer and other deadly illnesses. ${ }^{8,9}$ Therefore, these cultural beliefs wrapped together denote that the screening and diagnosis, as well as, treatment of cancer among culturally rooted groups pose a threat to high prevalence, morbidity and mortality rates of cancer. ${ }^{7}$

\section{B. The Importance of the Problem}

Understanding how various cultural beliefs influence individual or group decisions on the screening, diagnosis, and treatment processes, as well as, the various therapeutic platforms is vital for health interventionist to come up with culturally adjusted health education programs. For instance, a study by Pasick and Burke ${ }^{14}$ claimed that culturallyadjusted health intervention programs have a high chance of realizing positive results as opposed to general health intervention programs. For example, understanding the African cultural beliefs on cancer-susceptible foods is vital on understanding the impacts of such foods on the public health for that group, and come up with substitutable healthy foods that can be adopted instead of such cancerous foods. ${ }^{7}$ On the other hand, different cultural beliefs on cancer treatment, screening and diagnosis presents an opportunity to gather clinical practices adopted by the culturally-rooted groups that can be used as alternative treatments indicating that not all the beliefs have negative effects. ${ }^{7}$

The government and the health organizations would benefit from this problem since it sheds light on the platform that cultural beliefs afford cancer patients. Through different prevalence statistics, the government may determine the different groups that are prone to a high risk of cancer and use such data for increasing or introducing sufficient health support in such communities. For example, from the background research has identified that the blacks living in America are at high risks of prostate, lung, liver, and kidney cancer as compared to the whites. ${ }^{6}$ Such information is essential when planning for health care delivery and allocation of health care resources. Lastly, this problem opens an opportunity for identification of possible health care intervention strategies for cultural beliefs geared towards an efficient cancer treatment delivery for the public wellness.

\section{Interpretation of Findings}

A research Kleinman, ${ }^{8}$ by reported that culture has its own set of values, beliefs and practices that vary across different ethnic groups and these are key determinants for explaining disparities in cancer prevalence within the globe. For instance, the Africans have the highest chance in developing cancer from their beliefs set formulated from historical contexts of immigration, segregation and the fact that they belong to a minority group - they are part of the disadvantaged groups in America. ${ }^{8,}$ 9-12, 14 Such a culture factor has bestowed them with less power to seek for high income jobs which can give them potential purchasing power to access cancer health care. On the other hand, the fact such minorities are less advantaged over the years, it has become hard to access health education -indicting that the lack of income and relevant health education which results from cultural beliefs undermine personal willpower to live healthy especially for people in these groups. Therefore, these aspects can translate to silencing effect which may adversely affect cancer awareness. ${ }^{12}$ Nevertheless, these cultural aspects tied to beliefs make individuals less likely to adopt cancer-risk-reducing behaviors in addition to not seeking the right support, as well as, services when diagnosed with the illness. ${ }^{12,13}$

On a different note, research claims that the blacks and the Hispanics are the minority groups to believe that cardiovascular blood pressure is a causative factor to cancer. Also, they are the groups identified to believe that surgeries can cause lung cancer to intensify or spread. Additionally, beliefs such as fatalism and mistrust are so common among the blacks regarding their trust on doctors and professionals such as therapists. Also, such minority groups have mistrust with the modern medicine, treatment, and diagnosis techniques used in the modern cancer treatment. ${ }^{15}$ For instance, in the minority groups different beliefs about cancer treatment are unhealthy - some believe that surgery cannot be the appropriate treatment and that if an cancer patient is cut into the cancer, it spreads all over the body while other believe that treatments such as radiology would worsen the situation. ${ }^{15}$ Also, most cultures believe that some body parts are private and individuals should not talk of them, hence cervical cancer is prone to high prevalence among the African-American women. Breast or gynecological cancer among the black women may make them reluctant to disclose to practitioners for the fear of shame, such cases are reported when the symptoms or pain worsen or when the cancer is at a more vigilant level to handle. Again, such people with these beliefs are less willing to undergo for physical examinations to track the changes of the cancer activity. ${ }^{16}$

On a different note, individuals with cancer may feel isolation and believe that silence prevents stigma from 
family members, therefore, it explains why individuals with early symptoms of cancer fear to report them until the cases are out of hand or vigilant. A study by Mosher et al. ${ }^{6}$ added that the Africans have a high likelihood to believe that cancer treatment is a financial burden than the whites and this makes it hard to control cancer rates within this group. Therefore, it may concluded that these beliefs towards various aspects of cancer are unhealthy and present a chief obstacle for effective treatment of the disease leading to elevated prevalence of cancer in communities acculturated in these beliefs such as the blacks and the Asian-Americans. ${ }^{3}$

\section{MEASUREMENT ISSUES OF THE VARIABLES}

\section{A. Operationalization of the Variables}

In examining the effects of cultural beliefs on the prevalence of cancer, the cultural beliefs have been identified to escalate the prevalence of cancer as seen with the NCI report, the IARC survey and the individual research done on this field. Therefore, the "cultural beliefs" is treated as the independent variables while the "prevalence of cancer" the dependent variable. The concept of cancer prevalence has been operationalized in empirical research by the proportion of individuals with a given population living with the cancer or the cancer cases reported within a group at a given time. For instance, the vast empirical research on cancer within the American minority groups such as the blacks is identified as the proportion of African-Americans currently living with cancer or who had cancer within a particular timeframe.

The operationalization is valid as the government and other health organizations keeps updated health records for different illnesses, as well as, the immigrants for its minatory groups including the blacks, Asians, Indians, Mexicans and others. Therefore, this indicates that determining the ratio of blacks with cancer to their population is easy. On the other hand, given the fact that there has been a long period of racial segregation in America, it is possible to expect the type of beliefs identified by the research on the various minority groups tied to their culture. Lastly, operationalization of cultural beliefs has utilized the SelfRegulation Model $^{17}$ with the use of Illness Perception Questionnaire and Group-Based Medical Scales ${ }^{17}$ to assess how the different cultural beliefs influenced the decisions on treatment, diagnosis and screening, as well as, access to therapeutic sessions. Accordingly, these measurement platforms aim at quantifying personal and group-reported opinions, beliefs, views and though regarding cancer treatment modalities.

\section{CONCLUSION}

\section{A. Challenges}

Given the above analysis of the effects of cultural beliefs on prevalence there are various critical issues that need to be addressed upon future research. For instance, the cancer prevalence disparities appear to be insufficiently addressed by cultural issues along. Such disparities are critical to the progress of the fight against cancer. On the other hand, there is a need to research for opportunities in cancer disparities to help close the gap between the cancer health care between different socio-economic groups. One key aspect to address such a gap is through addressing of the oncology of cancer to identify the possible strategies or platforms that can be used to address the culturally-rooted factors affecting cancer treatment, adversely. Another current challenge in cancer health entails the public perceptions towards cancer diagnosis and treatment technology - in which most cultural groups view them as harmful and painful.

\section{B. Future Research Areas to Address}

In an attempt to address the above-identified issues, future researchers may aim at addressing how different beliefs affect each treatment modality, independently. Again, different types of cancer have been mentioned; breast; kidney, liver, lungs, prostate and gynecological cancers. Therefore, future research would address how each of these is influenced by beliefs from a particular cultural group. Lastly, addressing cost and quality issues related to cancer treatment and influence from cultural belies is a probable area to be explored - specifically to identify how cultural beliefs affect cost and quality of cancer health care.

\section{REFERENCES}

[1] Sloan PA, Donnelly MB, Schwartz RW, Sloan DA. Cancer pain assessment and management by house staff. Pain. 1996;67:475-81.

[2] Ferlay J, Bray F, Pisani P, Parkin DM: GLOBOCAN 2002: Cancer Incidence, Mortality and Prevalence Worldwide. IARC CancerBase No5. 2004, Lyon: IARC Press, http://www-dep.iarc.fr/.

[3] Anderson KO, Mendoza TR, Valero V, Richman SP, Russell C, Hurley J, et al. Minority cancer patients and their providers: Pain management attitudes and practice. Cancer. 2000;88:1929-38.

[4] U.S. Cancer Statistics Working Group. United States Cancer Statistics: 1999-2013 Incidence and Mortality Web-based Report. Atlanta (GA): Department of Health and Human Services, Centers for Disease Control and Prevention, and National Cancer Institute; 2016. Accessed November 23, 2016 http://www.cdc.gov/uscs.

[5] Haiman CA, Patterson N, Freedman ML, et al. Multiple regions within $8 \mathrm{q} 24$ independently affect risk for prostate cancer. Nature Genetics, 2007; 39(5); 638-644

[6] Mosher CE, DuHamel KN, Egert J, Smith MY. Self-efficacy for coping with cancer in a multiethnic sample of breast cancer patients: Associations with barriers to pain management and distress. Clin J Pain. 2010;26:227-34.

[7] Daher, M. Cultural beliefs and values in cancer patients. Annals of Oncology. 2013: Issue 23, (6) pp. 66-69.

[8] Kleinman A: Concepts and a model for the comparison of medical systems as cultural systems. Soc Sci Med. 2008, 12 (2B): 85-95.

[9] VanRyn M. Research on the provider contribution to race/ethnicity disparities in medical care. Med Care 2002; 40(1, Suppl)I140-I151.

[10] Im EO, Ko Y, Chee W. Symptom clusters among multiethnic groups of cancer patients with pain. Palliat Support Care. 2013;11:295-305.

[11] Chen CH, Tang ST, Chen CH. Meta-analysis of cultural differences in Western and Asian patient-perceived barriers to managing cancer pain. Palliat Med. 2012;26:206-21

[12] Hede K. Drilling down to the causes of racial disparities in lung cancer. J Natl Cancer Inst 2010;102:1385-1387.

[13] Jonnalagadda S, Bergamo C, Lin JJ, Lurslurchachai L, Diefenbach M, Smith C, Nelson JE, Wisnivesky JP. Beliefs and attitudes about lung cancer screening among smokers. Lung Cancer 2012;77:526-531. 
[14] Pasick RJ, Burke NJ: A critical review of theory in breast cancer screening promotion across cultures. Annu Rev Public Health. 2008, 29: 351-368.

[15] Thompson HS, Valdimarsdottir HB, Winkel G, Jandorf L, Redd W. The Group-Based Medical Mistrust Scale: psychometric properties and association with breast cancer screening. Prev Med 2004;38:209218.

[16] Carey LA, Perou CM, Livasy CA, et al. Race, breast cancer subtypes, and survival in the Carolina Breast Cancer Study. JAMA, 2006; 295(21); 2492-2502.

[17] Tam T. D, Al-Hareth A. K, Salha, B.A. Beliefs and attitudes about breast cancer and screening practices among Arab women living in Qatar: a cross-sectional study. BMC Women's Health2013. 